\title{
Likely Inflammatory Activity by PET
}

National Cancer Institute

\section{Source}

National Cancer Institute. Likely Inflammatory Activity by PET. NCI Thesaurus. Code C116102.

A finding of metabolic activity at the primary or nodal sites that is greater than liver uptake, but can be attributed to inflammation activity, as detected by FDG-PET. 